\title{
REMEDIATION ABILITY OF MELON GRASS IN A CRUDE OIL POLLUTED SOIL IN A TROPICAL REGION
}

Uwazie M.C. ${ }^{1}$, Obijiaku J.C. ${ }^{2}{ }^{凶}$, Onukwuli O.D. ${ }^{3}$, Babayemi A.K ${ }^{4}$, Umeuzuegbu J.C 5

${ }^{1}$ Department of Chemical Engineering, Imo State Polytechnic Umuagwo, Owerri, Nigeria

2 Department of Chemical Engineering, Federal University of Technology, Owerri, Nigeria

${ }^{3}$ Department of Chemical Engineering, Nnamdi Azikiwe University, Awka, Nigeria

4, 5 Department of Chemical Engineering, Chukwuemeka Odumegwu Ojukwu University, Uli. Nigeria

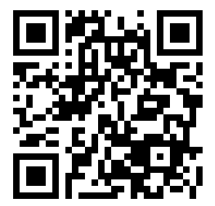

DOI: https://doi.org/10.29121/ijetmr.v7.i6.2020.527

Article Citation: Uwazie M.C., Obijiaku J.C., Onukwuli O.D., Babayemi A.K, and Umeuzuegbu J.C. (2020). REMEDIATION ABILITY OF MELON GRASS IN A CRUDE OIL POLLUTED SOIL IN A TROPICAL REGION. International Journal of Engineering Technologies and Management Research, 7(6), 89101.

https://doi.org/10.29121/ijetmr.v7 i6.2020.527

Published Date: 20 June 2020

Keywords:

Phytoremediation

Melon Grass

Crude Oil Polluted Soil

Physic-Chemical

Heavy Metals

\section{ABSTRACT}

The aim of this research was to study the effectiveness of a chosen grass (plant) in the remediation of soil contaminated by crude oil. Lemon grass was used in this study to remediate the polluted soil because of its perceived ability to do so. Five wooden boxes were fabricated, and $150 \mathrm{~kg}$ of soil were introduced to each of them. Four of the boxes were contaminated with different concentrations of crude oil, while two out of these four boxes were amended with cow dung. One of the boxes that served as control was neither contaminated nor amended. Lemon seeds were then planted in all the boxes and allowed to grow for the period of 12 weeks. Analysis were done at the beginning, during and after the plant growth in order to determine the rate of remediation, with particular interest in the TPH (total petroleum hydrocarbon), and few other heavy metals; copper, magnesium, aluminum. Two concentrations of contamination were artificially made (75g and $150 \mathrm{~g})$. The $\mathrm{TPH}, \mathrm{Pb}, \mathrm{Zn}$ and $\mathrm{Ni}$ contents of the $75 \mathrm{~g}$ crude oil contaminated soil were $6.784 \mu \mathrm{g} / \mathrm{g}$, $0.485 \mathrm{mg} / \mathrm{kg}, 0.867 \mathrm{mg} / \mathrm{kg}$ and $0.030 \mathrm{mg} / \mathrm{kg}$ respectively. It reduced to $6.356 \mu \mathrm{g} / \mathrm{g}, 0.058 \mathrm{mg} / \mathrm{kg}, 0.560 \mathrm{mg} / \mathrm{kg}$ and $0.043 \mathrm{mg} / \mathrm{kg}$ respectively after remediation. The TPHPb, $\mathrm{Zn}$ and Nicontents of the $150 \mathrm{~g}$ crude oil contaminated soil were $9.109 \mu \mathrm{g} / \mathrm{g}, 0.699 \mathrm{mg} / \mathrm{kg}, 0.851 \mathrm{mg} / \mathrm{kg}$ and $0.032 \mathrm{mg} / \mathrm{kg}$ respectively. It reduced $7.713 \mu \mathrm{g} / \mathrm{g}, 0.059 \mathrm{mg} / \mathrm{kg}, 0.506 \mathrm{mg} / \mathrm{kg}$ and $0.057 \mathrm{mg} / \mathrm{kg}$ respectively after remediation. An interesting observation was that, amending the contaminated soil with cow dung gave a favourable result in remediating the contaminants. It took about 80 months to remediate TPH to an acceptable level of $0.013 \mu \mathrm{g} / \mathrm{g}$, but over 150 months to do the same for an unamended soil. This finding can be applicable in the estimation of time requirement for the remediation of crude oil polluted soil by concerned stake holders.

\section{INTRODUCTION}

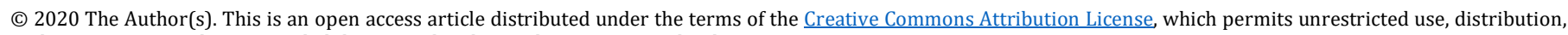
and reproduction in any medium, provided the original author and source are credited. 
Remediation Ability of Melon Grass in A Crude Oil Polluted Soil in A Tropical Region

Finding solutions to oil polluted sites (soil) has always been the subject of several studies (Leahy and Colwell, 1990). A wide range of remediation measures have been preferred with the aim of offering solution to the damages caused by crude oil on nature of the soil and its physiochemical characteristics. Over the decades, the biological methods of cleaning-up the environment have received much attention. This is because of its potential to reduce, detoxify and mineralize chemical pollution, restoring chemical balance at low cost. Phytoremediation is characterized by lots of advantages such as its cost effectiveness, environmental friendliness, simplicity in technology, conservation of soil texture and properties and its ability to produce harmless end products. This is contrary to other physical and chemical treatment methods whose limitations include; transfer of pollutants from one place/phase to another, being a complex technology and expensive to implement at full scale (Vidali, 2001). Due to the limitations of the physiochemical technologies stated above, great deal of literature has reported that phytoremediation methods are alternative and/or supplements to these methods.

Phytoremediation is a non-destructive, cost-effective in-situ technology that helps in a great measure to cleanup contaminated soils. If contamination is caused by petroleum hydrocarbons, plants can enhance microbial degradation of the contaminant in the rhizosphere. The potential for success of this technology for the tropics is high due to the prevailing climatic conditions enhancing plant growth and stimulating microbial activity.

Plants can render harmless, extract or stabilize a contaminant in soil, thereby making the contaminant(s) unavailable for other organisms and reducing environmental hazards. Mechanism of this technology called phytoremediation depends on the type of contaminant, bioavailability and soil properties.

During phytoremediation of petroleum-contaminated sites, harmful components can be metabolized by plant enzymes in the plant or in the soil. The mechanism believed to be responsible for most of the degradation of petroleum hydrocarbons in vegetated soil is the stimulation of growth and activity of degrading micro-organisms in the rhizophere. Because of root penetration, soil aggregates are loosened and oxygen supply in the soil increases. Oxygen is a decisive factor for the growth and activity of aerobics soil micro-organism and in the initial degradation step of a number of chemical compounds such as aromatics. Microbial activity also is enhanced by a number of substrates provided in the rhizosphere such as plant-derived enzymes, vitamins, hormones and low molecular carbons sources which promote microbial growth. Having a multiple ramified root system, grasses are mostly considered to be particularly suitable for phytoremediation because they offer an increased rhizosphere with a greater room for microbial activity and growth. In the other hand, the roots of legumes usually are not as ramified but usually reach deeper soil layers than grasses and thus take effect on deeper located contaminants. However, little is known about tropical species that will give an excellent result in the clean-up of oil contamination. The screening of plant species for their ability to grow and establish in contaminated soil is one of the first steps in the selection of species for phytoremediation in the tropics, followed by the evaluation of their influence on the degradation of petroleum hydrocarbons in soil.

This work selected a legume plant commonly found in abundance in the eastern parts of Nigeria (OwerriWest of Imo State), for screening for the purpose of phytoremediation in a country like ours where environmental contamination with petroleum-based products occur frequently.

The seeds of the plant were sown in different culture set ups as well as the control medium. The seedling emergence were monitored and data collected based on the roots, shoots and leave performance from the first day to a minimum of 90 days. The data were used to run analysis with the help of some known software. Tables and graphs were generated and arrayed to ease understanding of the performance of the process.

\section{MATERIALS AND METHODS}

\subsection{MATERIALS}

\subsubsection{SAMPLE/MATERIAL COLLECTION}

The soil sample used for the experiments was collected within Umuanunu Community in Obinze Town located inOwerri-West L.G.A of Imo State, and the melon seedlings used for the work was also purchased from same locality. The soil sample was collected from a fertile farmland location of the village communal farm area, with a large nylon bag and well labeled before being delivered to the laboratory for the laboratory tests.

\subsubsection{SOIL SAMPLE/MATERIAL PREPARATION}


Uwazie M.C., Obijiaku J.C., Onukwuli O.D., Babayemi A.K, and Umeuzuegbu J.C

The soil sample was superficially dried for two days after which it was further meshed and then sieved through $2 \mathrm{~mm}$ mesh size. The sieved soil samples were then used for the laboratory analysis.

Thirteen soil sample groups, each weighing $7.5 \mathrm{~kg}$ were measured out and separated for the experimental purpose. One of the groups was reserved as the control sample, while the other twelve were further divided into four groups of three categories, which were all artificially contaminated with petroleum to concentrations of 1 $\mathrm{w} / \mathrm{w} \%$ and $2 \mathrm{w} / \mathrm{w} \%$. Again, to the pair of the polluted samples, one of each group were further enriched with organic manure (cow dung), while the others were not. This was basically to check the effect of enrichment on the polluted soil, in the phytoremediation of the soil. In all, a total of thirteen soil sample groups (including the control sample) were prepared for the phytoremediation experiments.

\subsection{METHODS}

\subsubsection{EXPERIMENTAL PROCEDURE}

The phytoremediation study took place from the month of August to October 2017. The treatment was subdivided into four groups. Each of the treatment groups 1-4 constitutes three (3) replicate treatments. The only common proportion of all was the petroleum contaminated soil of $7.5 \mathrm{~kg}$. The target was to find out how different levels of contamination and enrichment would affect the degradation of a petroleum contaminated soil. The objective of the variation in the treatment levels was to investigate the most appropriate quantity of each treatment option that will give the best remediating result.

The soil set-up/grouping for the remediation study is as follows:

Group B: The three constituent replicates in this group were made up of $7.5 \mathrm{~kg}$ of soil $+75 \mathrm{~g}$ of crude oil $+150 \mathrm{~g}$ cow dung, each of which were analyzed at intervals of approximately six weeks during the twelve-week study period.

Group C: The three constituent replicates in this group were made up of $7.5 \mathrm{~kg}$ of soil $+150 \mathrm{~g}$ of crude oil $+150 \mathrm{~g}$ cow dung, each of which were analyzed at intervals of approximately six weeks during the twelve-week remediation study.

Group D: The three constituent replicates in this group were made up of $7.5 \mathrm{~kg}$ of soil $+75 \mathrm{~g}$ of crude oil, each of which were analyzed at approximately six-week intervals for the period of twelve weeks.

Group E: The three constituent replicates in this group were made up of $7.5 \mathrm{~kg}$ of soil $+150 \mathrm{~g}$ of crude oil, each of which were analyzed at intervals of approximately six weeks for the period of twelve weeks.

It is worthy of mention that several studies have demonstrated the necessity of oxygen in phytoremediation of oil-contaminated soil, hence all the replicates were supplied with little watering and exposed to oxygen by milled tilling in order to facilitate degradation.

Table 2.1 shows how the experiment was divided into four treatment groups; each treatment had $7.5 \mathrm{~kg}$ of soil plus different proportions (75g or $150 \mathrm{~g}$ ) of crude oil and then either with or without organic manure.

Table 2.1: Experimental design for the phytoremediation study

\begin{tabular}{|c|c|}
\hline Options & Treatment/enrichment \\
\hline Group B & $7.5 \mathrm{~kg}$ soil $+75 \mathrm{~g}$ crude oil $+150 \mathrm{~g}$ cow dung \\
\hline Group C & $7.5 \mathrm{~kg}$ soil $+150 \mathrm{~g}$ crude oil $+150 \mathrm{~g}$ cow dung \\
\hline Group D & $7.5 \mathrm{~kg}$ soil $+75 \mathrm{~g}$ crude oil \\
\hline Group E & $7.5 \mathrm{~kg}$ soil $+150 \mathrm{~g}$ crude oil \\
\hline
\end{tabular}

The experimental layout is shown in Table 2.2 below;

Table 2.2: Experimental Layout

\begin{tabular}{|l|c|c|c|c|}
\hline & \multicolumn{4}{|c|}{ Treatment Groups } \\
\hline Treatment number & $\mathrm{B}$ & $\mathrm{C}$ & $\mathrm{D}$ & $\mathrm{E}$ \\
\hline 1 (Before planting) & $\mathrm{S}_{\mathrm{B} 1}$ & $\mathrm{~S}_{\mathrm{C} 1}$ & $\mathrm{~S}_{\mathrm{D} 1}$ & $\mathrm{~S}_{\mathrm{E} 1}$ \\
\hline 2 (Within planting period) & $\mathrm{S}_{\mathrm{B} 2}$ & $\mathrm{~S}_{\mathrm{C} 2}$ & $\mathrm{~S}_{\mathrm{D} 2}$ & $\mathrm{~S}_{\mathrm{E} 2}$ \\
\hline 3 (After planting) & $\mathrm{S}_{\mathrm{B} 3}$ & $\mathrm{~S}_{\mathrm{C} 3}$ & $\mathrm{~S}_{\mathrm{D} 3}$ & $\mathrm{~S}_{\mathrm{E} 3}$ \\
\hline
\end{tabular}

Where:

$\mathrm{S}_{\mathrm{B} 1-} \quad 7.5 \mathrm{~kg}$ soil $+75 \mathrm{~g}$ crude oil $+150 \mathrm{~g}$ cow dung (Before planting)

International Journal of Engineering Technologies and Management Research 
Remediation Ability of Melon Grass in A Crude Oil Polluted Soil in A Tropical Region

$\mathrm{S}_{\mathrm{B} 2-} \quad \quad 7.5 \mathrm{~kg}$ soil $+75 \mathrm{~g}$ crude oil $+150 \mathrm{~g}$ cow dung (Within planting period)

$\mathrm{S}_{\mathrm{B} 3}-\quad 7.5 \mathrm{~kg}$ soil $+75 \mathrm{~g}$ crude oil $+150 \mathrm{~g}$ cow dung (After planting)

$\mathrm{S}_{\mathrm{C} 1}-\quad 7.5 \mathrm{~kg}$ soil $+150 \mathrm{~g}$ crude oil $+150 \mathrm{~g}$ cow dung (Before planting)

$\mathrm{S}_{\mathrm{C} 2}-\quad 7.5 \mathrm{~kg}$ soil $+150 \mathrm{~g}$ crude oil $+150 \mathrm{~g}$ cow dung (Within planting period)

$\mathrm{S}_{\mathrm{C} 3}-\quad 7.5 \mathrm{~kg}$ soil $+150 \mathrm{~g}$ crude oil $+150 \mathrm{~g}$ cow dung (After planting)

$\mathrm{S}_{\mathrm{D} 1^{-}} \quad 7.5 \mathrm{~kg}$ soil $+75 \mathrm{~g}$ crude oil (Before planting)

$\mathrm{S}_{\mathrm{D} 2-} \quad 7.5 \mathrm{~kg}$ soil $+75 \mathrm{~g}$ crude oil (Within planting period)

$\mathrm{S}_{\mathrm{D} 3^{-}} \quad$ 7.5kg soil $+75 \mathrm{~g}$ crude oil (After planting)

$\mathrm{S}_{\mathrm{E} 1}-\quad 7.5 \mathrm{~kg}$ soil $+150 \mathrm{~g}$ crude oil (Before planting)

$\mathrm{S}_{\mathrm{E} 2}-\quad 7.5 \mathrm{~kg}$ soil $+150 \mathrm{~g}$ crude oil (Within planting period)

$\mathrm{S}_{\mathrm{E} 3}-\quad \quad \quad 7.5 \mathrm{~kg}$ soil $+150 \mathrm{~g}$ crude oil (After planting)

This research work was conducted for approximately twelve weeks, during which soil and plant samples were taken to the laboratory for analysis at six weeks interval.

\section{RESULTS AND DISCUSSION}

\subsection{RESULTS PRESENTATION}

Results of the laboratory tests conducted on the soil and plant samples obtainedare presented below:

Table 3.1: Physico-chemical parameters for unpolluted soil sample

\begin{tabular}{|l|l|c|c|c|c|}
\hline $\mathrm{S} / \mathrm{N}$ & Parameter & Run 1 & Run 2 & Run 3 & Average \\
\hline 1 & $\mathrm{pH}$ & 6.92 & 6.90 & 6.91 & 6.91 \\
\hline 2 & Moisture content, \% & 1.87 & 2.54 & 2.10 & 2.17 \\
\hline 3 & Ash content, \% & 94.02 & 93.68 & 94.00 & 93.90 \\
\hline 4 & Organic matter, \% & 5.98 & 5.47 & 5.88 & 5.78 \\
\hline 5 & TOC, \% & 1.60 & 1.70 & 1.60 & 1.63 \\
\hline 6 & TPH, $\mu \mathrm{g} / \mathrm{g}$ & 0.014 & 0.010 & 0.016 & 0.013 \\
\hline 7 & Nitrate-Nitrogen, $\mathrm{mg} / \mathrm{kg} \mathrm{NO}{ }_{3}-\mathrm{N}$ & 11.20 & 10.94 & 10.53 & 10.89 \\
\hline 8 & Ammonia- Nitrogen, $\mathrm{mg} / \mathrm{kg} \mathrm{NH}_{3}-\mathrm{N}$ & 3.50 & 3.39 & 3.44 & 3.44 \\
\hline 9 & Potassium, $\mathrm{mg} / \mathrm{kg} \mathrm{K}$ & 40.00 & 45.00 & 50.00 & 45.00 \\
\hline 10 & Calcium, $\mathrm{mg} / \mathrm{kg} \mathrm{Ca}$ & 12.62 & 12.66 & 11.30 & 12.19 \\
\hline 11 & Nickel, $\mathrm{mg} / \mathrm{kg} \mathrm{Ni}$ & 0.15 & 0.13 & 0.14 & 0.14 \\
\hline 12 & Copper, $\mathrm{mg} / \mathrm{kg} \mathrm{Cu}$ & 0.17 & 0.17 & 0.16 & 0.17 \\
\hline 13 & Zinc, $\mathrm{mg} / \mathrm{kg} \mathrm{Zn}$ & 1.57 & 1.58 & 1.62 & 1.60 \\
\hline 14 & Lead, $\mathrm{mg} / \mathrm{kg} \mathrm{Pb}$ & 2.06 & 2.04 & 1.83 & 1.98 \\
\hline
\end{tabular}

Table 3.2: Textural class of unpolluted soil sample

\begin{tabular}{|l|l|c|c|}
\hline S/N & Textural Class & Mesh Size $(\mathrm{Mm})$ & Soil Size (\%) \\
\hline 1 & \multirow{2}{*}{ Pebble Stone } & 4.750 & - \\
\cline { 3 - 4 } & & 3.350 & - \\
\hline 2 & Very Coarse Sand & 1.180 & 0.61 \\
\hline 3 & Coarse Sand & 1.000 & 0.87 \\
\hline 4 & Medium Coarse Sand & 0.425 & 31.44 \\
\cline { 3 - 4 } & & 0.300 & 18.86 \\
\hline \multirow{2}{*}{5} & \multirow{2}{*}{ Fine Sand } & 0.212 & 30.90 \\
\cline { 3 - 4 } & & 0.125 & 8.96 \\
\cline { 3 - 4 } & & 0.106 & 2.98 \\
\hline 6 & \multirow{2}{*}{ Very Fine Sand } & 0.075 & 2.13 \\
\cline { 3 - 4 } & & 0.053 & 0.05 \\
\hline 7 & Silt & $<0.053$ & 3.20 \\
\hline
\end{tabular}


Uwazie M.C., Obijiaku J.C., Onukwuli O.D., Babayemi A.K, and Umeuzuegbu J.C

3.1.1. SOIL ANALYSIS BEFORE PLANTING, 18 ${ }^{\text {TH }}$ AUGUST 2017

Table 3.3: Physico-chemical parameters for polluted soil sample (amended)

\begin{tabular}{|c|c|c|c|c|c|c|c|c|c|}
\hline \multirow[t]{2}{*}{$\mathrm{S} / \mathrm{N}$} & \multirow[t]{2}{*}{ Parameter } & \multicolumn{4}{|c|}{$\begin{array}{l}\text { B (7.5kg Soil polluted with 75g crude oil } \\
+150 \mathrm{~g} \text { Cow dung) }\end{array}$} & \multicolumn{4}{|c|}{$\begin{array}{l}\text { C (7.5kg Soil polluted with } 150 \mathrm{~g} \text { crude oil } \\
+150 \mathrm{~g} \text { Cow dung) }\end{array}$} \\
\hline & & $\mathrm{B}_{1}$ & $\mathrm{~B}_{2}$ & $\mathrm{~B}_{3}$ & Average & $\mathrm{C}_{1}$ & $\mathrm{C}_{2}$ & $\mathrm{C}_{3}$ & Average \\
\hline 1 & $\mathrm{pH}$ & 7.07 & 7.08 & 7.05 & 7.07 & 7.18 & 7.21 & 7.22 & 7.20 \\
\hline 2 & $\begin{array}{l}\text { Moisture content, } \\
\%\end{array}$ & 15.46 & 15.40 & 15.67 & 15.51 & 13.84 & 13.84 & 13.37 & 13.68 \\
\hline 3 & Ash content, \% & 79.02 & 79.24 & 80.44 & 79.57 & 81.23 & 82.85 & 81.85 & 81.98 \\
\hline 4 & Organic matter, \% & 20.98 & 20.76 & 19.56 & 20.43 & 22.73 & 24.47 & 23.75 & 23.65 \\
\hline 5 & TOC, $\%$ & 5.40 & 5.70 & 5.60 & 5.57 & 5.84 & 5.93 & 5.79 & 5.85 \\
\hline 6 & $\mathrm{TPH}, \mu \mathrm{g} / \mathrm{g}$ & 6.783 & 6.785 & 6.785 & 6.784 & 9.394 & 9.175 & 8.757 & 9.109 \\
\hline 7 & $\begin{array}{l}\text { Nitrate-Nitrogen, } \\
\mathrm{mg} / \mathrm{kg} \mathrm{NO}-\mathrm{N}\end{array}$ & 12.50 & 11.86 & 11.95 & 12.10 & 11.95 & 11.37 & 11.75 & 11.69 \\
\hline 8 & $\begin{array}{l}\text { Ammonia- } \\
\text { Nitrogen, mg/kg } \\
\mathrm{NH}_{3}-\mathrm{N}\end{array}$ & 3.90 & 3.84 & 3.88 & 3.87 & 3.96 & 3.92 & 3.91 & 3.93 \\
\hline 9 & $\begin{array}{l}\text { Potassium, mg/kg } \\
\mathrm{K}\end{array}$ & 46.00 & 47.00 & 47.00 & 46.67 & 44.58 & 43.74 & 44.57 & 44.30 \\
\hline 10 & Calcium, mg/kg Ca & 11.56 & 11.34 & 11.48 & 11.46 & 10.74 & 11.05 & 11.17 & 10.99 \\
\hline 11 & Nickel, mg/kg Ni & 0.029 & 0.029 & 0.031 & 0.030 & 0.032 & 0.030 & 0.034 & 0.032 \\
\hline 12 & Copper, mg/kg Cu & 3.186 & 3.218 & 3.120 & 3.175 & 4.947 & 4.853 & 4.825 & 4.875 \\
\hline 13 & Zinc, $\mathrm{mg} / \mathrm{kg} \mathrm{Zn}$ & 0.984 & 0.823 & 0.793 & 0.867 & 0.904 & 0.804 & 0.846 & 0.851 \\
\hline 14 & Lead, mg/kg Pb & 0.456 & 0.472 & 0.512 & $0.48 \mathrm{~S}$ & 0.674 & 0.695 & 0.729 & 0.699 \\
\hline
\end{tabular}

Table 3.4: Physico-chemical parameters for polluted soil sample (unamended)

\begin{tabular}{|c|c|c|c|c|c|c|c|c|c|}
\hline \multirow[t]{2}{*}{$\mathrm{S} / \mathrm{N}$} & \multirow[t]{2}{*}{ Parameter } & \multicolumn{4}{|c|}{$\begin{array}{l}\text { D (7.5kg Soil polluted with 75g crude } \\
\text { oil) }\end{array}$} & \multicolumn{4}{|c|}{$\begin{array}{l}\text { E (7.5kg Soil polluted with } 150 \mathrm{~g} \text { crude } \\
\text { oil) }\end{array}$} \\
\hline & & $\mathrm{D}_{1}$ & $\mathrm{D}_{2}$ & $\mathrm{D}_{3}$ & Average & $\mathrm{E}_{1}$ & $\mathrm{E}_{2}$ & $\mathrm{E}_{3}$ & Average \\
\hline 1 & $\mathrm{pH}$ & 7.24 & 7.28 & 7.26 & 7.26 & 7.43 & 7.43 & 7.42 & 7.43 \\
\hline 2 & Moisture content, $\%$ & 14.53 & 14.41 & 14.26 & 14.40 & 11.16 & 11.20 & 11.11 & 11.16 \\
\hline 3 & Ash content, $\%$ & 81.02 & 81.94 & 82.34 & 81.77 & 84.85 & 84.90 & 84.93 & 84.89 \\
\hline 4 & Organic matter, \% & 14.48 & 13.96 & 14.26 & 14.23 & 15.15 & 15.10 & 15.07 & 15.11 \\
\hline 5 & TOC, $\%$ & 6.46 & 7.17 & 7.04 & 6.89 & 8.03 & 8.15 & 9.02 & 8.40 \\
\hline 6 & $\mathrm{TPH}, \mu \mathrm{g} / \mathrm{g}$ & 7.247 & 7.937 & 7.573 & 7.59 & 9.95 & 10.05 & 10.12 & 10.04 \\
\hline 7 & $\begin{array}{l}\text { Nitrate-Nitrogen, } \mathrm{mg} / \mathrm{kg} \\
\mathrm{NO}_{3}-\mathrm{N}\end{array}$ & 11.50 & 11.47 & 11.25 & 14.41 & 11.22 & 11.04 & 11.24 & 11.17 \\
\hline 8 & $\begin{array}{l}\text { Ammonia- Nitrogen, } \\
\mathrm{mg} / \mathrm{kg} \mathrm{NH}-\mathrm{N}\end{array}$ & 3.49 & 3.72 & 3.81 & 3.67 & 3.74 & 3.72 & 3.79 & 3.75 \\
\hline 9 & Potassium, mg/kg K & 45.17 & 44.63 & 45.73 & 45.18 & 44.26 & 42.74 & 43.16 & 43.39 \\
\hline 10 & Calcium, mg/kg Ca & 11.36 & 11.28 & 11.25 & 11.30 & 11.27 & 11.28 & 11.15 & 11.23 \\
\hline 11 & Nickel, mg/kg Ni & 0.005 & 0.003 & 0.002 & 0.003 & 0.000 & 0.000 & 0.000 & 0.000 \\
\hline 12 & Copper, mg/kg Cu & 3.172 & 3.203 & 3.120 & 3.165 & 0.410 & 0.408 & 0.410 & 0.409 \\
\hline 13 & Zinc, mg/kg Zn & 0.843 & 0.853 & 0.786 & 0.827 & 0.850 & 0.854 & 0.852 & 0.852 \\
\hline 14 & Lead, mg/kg Pb & 0.346 & 0.329 & 0.362 & 0.436 & 0.404 & 0.402 & 0.398 & 0.401 \\
\hline
\end{tabular}


Remediation Ability of Melon Grass in A Crude Oil Polluted Soil in A Tropical Region 3.1.2. SOIL ANALYSIS WITHIN PLANTING PERIOD, $26^{\text {TH }}$ SEPTEMBER 2017

Table 3.5: Physico-chemical parameters for polluted soil sample (amended)

\begin{tabular}{|c|c|c|c|c|c|c|c|c|c|}
\hline \multirow[t]{2}{*}{$\mathrm{S} / \mathrm{N}$} & \multirow[t]{2}{*}{ Parameter } & \multicolumn{4}{|c|}{$\begin{array}{l}\text { B (7.5kg Soil polluted with 75g crude oil } \\
+150 \mathrm{~g} \text { Cow dung) }\end{array}$} & \multicolumn{4}{|c|}{$\begin{array}{l}\text { C (7.5kg Soil polluted with } 150 \mathrm{~g} \text { crude oil } \\
+150 \mathrm{~g} \text { Cow dung) }\end{array}$} \\
\hline & & $\mathrm{B}_{1}$ & $\mathrm{~B}_{2}$ & $\mathrm{~B}_{3}$ & Average & $\mathrm{C}_{1}$ & $\mathrm{C}_{2}$ & $\mathrm{C}_{3}$ & Average \\
\hline 1 & $\mathrm{pH}$ & 6.33 & 6.37 & 6.35 & 6.35 & 6.55 & 6.61 & 6.59 & 6.58 \\
\hline 2 & $\begin{array}{l}\text { Moisture content, } \\
\%\end{array}$ & 12.64 & 13.47 & 12.83 & 12.98 & 12.61 & 12.44 & 12.23 & 12.43 \\
\hline 3 & Ash content, \% & 72.53 & 71.46 & 72.07 & 72.02 & 75.26 & 74.93 & 74.48 & 74.89 \\
\hline 4 & Organic matter, \% & 16.46 & 15.43 & 15.38 & 15.76 & 20.04 & 21.46 & 20.92 & 20.81 \\
\hline 5 & TOC, $\%$ & 5.21 & 5.27 & 5.18 & 5.22 & 5.42 & 5.38 & 5.29 & 5.36 \\
\hline 6 & $\mathrm{TPH}, \mu \mathrm{g} / \mathrm{g}$ & 6.493 & 6.515 & 6.481 & 6.500 & 8.419 & 8.673 & 8.236 & 8.443 \\
\hline 7 & $\begin{array}{l}\text { Nitrate-Nitrogen, } \\
\mathrm{mg} / \mathrm{kg} \mathrm{NO}-\mathrm{N}\end{array}$ & 11.24 & 11.28 & 11.07 & 11.20 & 11.07 & 11.11 & 10.98 & 11.05 \\
\hline 8 & $\begin{array}{l}\text { Ammonia- } \\
\text { Nitrogen, mg/kg } \\
\mathrm{NH}_{3}-\mathrm{N}\end{array}$ & 3.66 & 3.64 & 3.67 & 3.66 & 3.58 & 3.44 & 3.51 & 3.51 \\
\hline 9 & $\begin{array}{l}\text { Potassium, } \mathrm{mg} / \mathrm{kg} \\
\mathrm{K}\end{array}$ & 23.84 & 25.29 & 22.22 & 23.78 & 18.94 & 20.17 & 19.73 & 19.61 \\
\hline 10 & Calcium, mg/kg Ca & 11.13 & 10.84 & 10.88 & 10.95 & 10.67 & 11.80 & 11.72 & 11.40 \\
\hline 11 & Nickel, mg/kg Ni & 0.025 & 0.035 & 0.033 & 0.031 & 0.053 & 0.061 & 0.057 & 0.057 \\
\hline 12 & Copper, mg/kg Cu & 1.763 & 1.484 & 1.629 & 1.625 & 1.903 & 2.128 & 2.064 & 2.032 \\
\hline 13 & Zinc, $\mathrm{mg} / \mathrm{kg} \mathrm{Zn}$ & 0.784 & 0.693 & 0.663 & 0.713 & 0.746 & 0.772 & 0.809 & 0.776 \\
\hline 14 & Lead, mg/kg Pb & 0.127 & 0.174 & 0.211 & 0.171 & 0.244 & 0.219 & 0.259 & 0.241 \\
\hline
\end{tabular}

Table 3.6: Physico-chemical parameters for polluted soil sample (unamended)

\begin{tabular}{|c|c|c|c|c|c|c|c|c|c|}
\hline \multirow[t]{2}{*}{$\mathrm{S} / \mathrm{N}$} & \multirow[t]{2}{*}{ Parameter } & \multicolumn{4}{|c|}{$\begin{array}{l}\text { D (7.5kg Soil polluted with } 75 g \text { crude } \\
\text { oil) }\end{array}$} & \multicolumn{4}{|c|}{$\begin{array}{l}\text { E (7.5kg Soil polluted with } 150 \mathrm{~g} \\
\text { crude oil) }\end{array}$} \\
\hline & & $\mathrm{D}_{1}$ & $\mathrm{D}_{2}$ & $\mathrm{D}_{3}$ & Average & $E_{1}$ & $\mathrm{E}_{2}$ & $\mathrm{E}_{3}$ & Average \\
\hline 1 & $\mathrm{pH}$ & 7.02 & 6.93 & 6.87 & 6.94 & 7.14 & 7.19 & 7.24 & 7.19 \\
\hline 2 & Moisture content, $\%$ & 12.35 & 11.84 & 12.07 & 12.09 & 11.11 & 11.05 & 10.81 & 10.99 \\
\hline 3 & Ash content, \% & 73.83 & 75.28 & 72.37 & 73.83 & 77.38 & 74.92 & 76.35 & 76.22 \\
\hline 4 & Organic matter, $\%$ & 13.83 & 13.73 & 12.96 & 13.51 & 15.78 & 15.64 & 15.47 & 15.72 \\
\hline 5 & TOC, $\%$ & 6.09 & 5.67 & 5.95 & 5.90 & 7.58 & 7.30 & 8.06 & 7.65 \\
\hline 6 & $\mathrm{TPH}, \mu \mathrm{g} / \mathrm{g}$ & 6.37 & 7.47 & 6.99 & 6.94 & 7.89 & 7.92 & 8.11 & 7.97 \\
\hline 7 & $\begin{array}{l}\text { Nitrate-Nitrogen, } \mathrm{mg} / \mathrm{kg} \\
\mathrm{NO}_{3}-\mathrm{N}\end{array}$ & 11.64 & 11.28 & 11.73 & 11.55 & 11.19 & 11.27 & 11.15 & 11.20 \\
\hline 8 & $\begin{array}{l}\text { Ammonia- Nitrogen, } \\
\mathrm{mg} / \mathrm{kg} \mathrm{NH}-\mathrm{N}\end{array}$ & 3.56 & 3.47 & 3.73 & 3.59 & 3.28 & 3.52 & 3.51 & 3.44 \\
\hline 9 & Potassium, mg/kg K & 18.754 & 16.947 & 21.196 & 18.966 & 24.67 & 22.76 & 19.85 & 22.43 \\
\hline 10 & Calcium, mg/kg Ca & 10.63 & 10.46 & 11.04 & 10.71 & 11.02 & 11.17 & 11.21 & 11.13 \\
\hline 11 & Nickel, mg/kg Ni & 0.043 & 0.051 & 0.049 & 0.0477 & 0.018 & 0.030 & 0.026 & 0.025 \\
\hline 12 & Copper, mg/kg Cu & 1.532 & 2.572 & 3.043 & 2.382 & 2.462 & 2.368 & 3.115 & 2.648 \\
\hline 13 & Zinc, mg/kg Zn & 0.653 & 0.715 & 0.863 & 0.744 & 0.780 & 0.556 & 0.704 & 0.680 \\
\hline 14 & Lead, mg/kg Pb & 0.164 & 0.229 & 0.172 & 0.188 & 0.207 & 0.184 & 0.191 & 0.194 \\
\hline
\end{tabular}


Uwazie M.C., Obijiaku J.C., Onukwuli O.D., Babayemi A.K, and Umeuzuegbu J.C

3.1.3. SOIL ANALYSIS AFTER PLANTING, 24TH OCTOBER 2017

Table 3.7: Physico-chemical parameters for polluted soil sample (amended)

\begin{tabular}{|c|c|c|c|c|c|c|c|c|c|}
\hline \multirow[t]{2}{*}{$\mathrm{S} / \mathrm{N}$} & \multirow[t]{2}{*}{ Parameter } & \multicolumn{4}{|c|}{$\begin{array}{l}\text { B (7.5kg Soil polluted with 75g crude oil } \\
+150 \mathrm{~g} \text { Cow dung) }\end{array}$} & \multicolumn{4}{|c|}{$\begin{array}{l}\text { C (7.5kg Soil polluted with } 150 \mathrm{~g} \text { crude oil } \\
+150 \mathrm{~g} \text { Cow dung) }\end{array}$} \\
\hline & & $\mathrm{B}_{1}$ & $\mathrm{~B}_{2}$ & $\mathrm{~B}_{3}$ & Average & $\mathrm{C}_{1}$ & $\mathrm{C}_{2}$ & $\mathrm{C}_{3}$ & Average \\
\hline 1 & $\mathrm{pH}$ & 6.17 & 6.04 & 6.11 & 6.11 & 6.28 & 6.24 & 6.19 & 6.24 \\
\hline 2 & $\begin{array}{l}\text { Moisture content, } \\
\%\end{array}$ & 10.74 & 11.46 & 10.72 & 10.97 & 10.27 & 11.36 & 10.38 & 10.67 \\
\hline 3 & Ash content, \% & 69.38 & 66.84 & 64.44 & 66.89 & 71.37 & 70.83 & 71.34 & 71.18 \\
\hline 4 & Organic matter, $\%$ & 16.84 & 15.74 & 14.95 & 15.84 & 17.37 & 16.38 & 15.59 & 16.45 \\
\hline 5 & TOC, $\%$ & 5.18 & 5.04 & 4.75 & 4.99 & 5.36 & 5.37 & 3.49 & 14.22 \\
\hline 6 & $\mathrm{TPH}, \mu \mathrm{g} / \mathrm{g}$ & 6.236 & 6.485 & 6.356 & 6.359 & 7.473 & 7.483 & 8.183 & 7.713 \\
\hline 7 & $\begin{array}{l}\text { Nitrate-Nitrogen, } \\
\mathrm{mg} / \mathrm{kg} \mathrm{NO} \mathrm{N}_{3} \mathrm{~N}\end{array}$ & 11.17 & 10.86 & 11.04 & 10.022 & 11.03 & 10.73 & 10.82 & 10.86 \\
\hline 8 & $\begin{array}{l}\text { Ammonia- } \\
\text { Nitrogen, mg/kg } \\
\mathrm{NH}_{3}-\mathrm{N}\end{array}$ & 3.38 & 3.43 & 3.36 & 3.39 & 3.18 & 3.33 & 3.42 & 3.31 \\
\hline 9 & $\begin{array}{l}\text { Potassium, mg/kg } \\
\mathrm{K}\end{array}$ & 3.379 & 3.337 & 3.603 & 3.440 & 3.205 & 3.301 & 3.395 & 3.300 \\
\hline 10 & $\begin{array}{l}\text { Calcium, mg/kg } \\
\text { Ca }\end{array}$ & 10.26 & 10.73 & 10.29 & 10.43 & 10.00 & 10.05 & 10.02 & 10.02 \\
\hline 11 & Nickel, mg/kg Ni & 0.043 & 0.042 & 0.044 & 0.043 & 0.052 & 0.061 & 0.058 & 0.057 \\
\hline 12 & Copper, mg/kg Cu & 0.062 & 0.060 & 0.062 & 0.061 & 0.066 & 0.062 & 0.064 & 0.064 \\
\hline 13 & Zinc, mg/kg Zn & 0.565 & 0.568 & 0.546 & 0.560 & 0.502 & 0.497 & 0.518 & 0.506 \\
\hline 14 & Lead, mg/kg Pb & 0.0580 & 0.058 & 0.058 & 0.058 & 0.0572 & 0.059 & 0.062 & 0.059 \\
\hline
\end{tabular}

Table 3.8: Physico-chemical parameters for polluted soil sample (unamended)

\begin{tabular}{|c|c|c|c|c|c|c|c|c|c|}
\hline \multirow[t]{2}{*}{$\mathrm{S} / \mathrm{N}$} & \multirow[t]{2}{*}{ Parameter } & \multicolumn{4}{|c|}{$\begin{array}{l}\text { D (7.5kg Soil polluted with } 75 \mathrm{~g} \text { crude } \\
\text { oil) }\end{array}$} & \multicolumn{4}{|c|}{$\begin{array}{l}\text { E (7.5kg Soil polluted with } 150 \mathrm{~g} \text { crude } \\
\text { oil) }\end{array}$} \\
\hline & & $\mathrm{D}_{1}$ & $\mathrm{D}_{2}$ & $\mathrm{D}_{3}$ & Average & $\mathrm{E}_{1}$ & $\mathrm{E}_{2}$ & $\mathrm{E}_{3}$ & Average \\
\hline 1 & $\mathrm{pH}$ & 6.58 & 7.05 & 6.92 & 6.85 & 6.86 & 6.96 & 6.94 & 6.92 \\
\hline 2 & Moisture content, $\%$ & 10.92 & 10.37 & 11.83 & 11.04 & 11.52 & 10.94 & 10.78 & 11.08 \\
\hline 3 & Ash content, $\%$ & 65.73 & 67.03 & 66.37 & 66.38 & 67.03 & 67.59 & 68.84 & 67.82 \\
\hline 4 & Organic matter, $\%$ & 14.72 & 13.82 & 12.38 & 13.64 & 16.94 & 15.37 & 15.98 & 16.10 \\
\hline 5 & TOC, $\%$ & 4.72 & 4.63 & 4.44 & 4.60 & 5.12 & 5.03 & 4.95 & 5.03 \\
\hline 6 & $\mathrm{TPH}, \mu \mathrm{g} / \mathrm{g}$ & 6.537 & 6.836 & 6.756 & 6.710 & 6.936 & 6.904 & 7.059 & 6.966 \\
\hline 7 & $\begin{array}{l}\text { Nitrate-Nitrogen, } \mathrm{mg} / \mathrm{kg} \\
\mathrm{NO}_{3}-\mathrm{N}\end{array}$ & 11.03 & 10.78 & 10.65 & 10.82 & 10.83 & 10.62 & 10.91 & 10.79 \\
\hline 8 & $\begin{array}{l}\text { Ammonia- Nitrogen, } \\
\mathrm{mg} / \mathrm{kg} \mathrm{NH} \text {-N }\end{array}$ & 3.23 & 3.17 & 3.21 & 3.20 & 3.15 & 3.09 & 3.14 & 3.13 \\
\hline 9 & Potassium, mg/kg K & 8.103 & 7.937 & 8.073 & 8.038 & 12.783 & 12.750 & 12.762 & 12.765 \\
\hline 10 & Calcium, mg/kg Ca & 8.26 & 9.49 & 9.92 & 9.22 & 9.82 & 9.38 & 9.86 & 9.69 \\
\hline 11 & Nickel, mg/kg Ni & 0.033 & 0.038 & 0.037 & 0.036 & 0.021 & 0.021 & 0.026 & 0.023 \\
\hline 12 & Copper, mg/kg Cu & 0.483 & 0.488 & 0.464 & 0.478 & 0.578 & 0.569 & 0.581 & 0.576 \\
\hline 13 & Zinc, $\mathrm{mg} / \mathrm{kg} \mathrm{Zn}$ & 0.483 & 0.485 & 0.492 & 0.487 & 0.404 & 0.412 & 0.382 & 0.399 \\
\hline 14 & Lead, $\mathrm{mg} / \mathrm{kg} \mathrm{Pb}$ & 0.020 & 0.027 & 0.021 & 0.023 & 0.000 & 0.000 & 0.006 & 0.002 \\
\hline
\end{tabular}




\subsubsection{ANALYSIS ON PLANT}

Remediation Ability of Melon Grass in A Crude Oil Polluted Soil in A Tropical Region

Table 3.9: Physico-chemical parameters for control plant

\begin{tabular}{|l|l|c|c|c|c|}
\hline $\mathrm{S} / \mathrm{N}$ & Parameter & Run 1 & Run 2 & Run 3 & Average \\
\hline 1 & Moisture content, \% & 18.44 & 19.03 & 18.37 & 16.61 \\
\hline 2 & Ash content, \% & 12.87 & 11.97 & 12.06 & 12.30 \\
\hline 3 & Organic matter, \% & 87.68 & 88.73 & 87.28 & 87.91 \\
\hline 4 & Nitrate-Nitrogen, mg/kg NO ${ }_{3}-\mathrm{N}$ & 9.47 & 10.06 & 10.18 & 9.90 \\
\hline 5 & Potassium, mg/kg K & 28.46 & 31.17 & 29.03 & 29.55 \\
\hline 6 & Calcium, mg/kg Ca & 16.83 & 17.08 & 16.56 & 16.82 \\
\hline 7 & Nickel, mg/kg Ni & 1.652 & 1.652 & 1.652 & 1.652 \\
\hline 8 & Mercury, mg/kg Hg & 0.988 & 0.988 & 0.974 & 0.983 \\
\hline 9 & Cadmium, mg/kg Cd & 0.072 & 0.075 & 0.072 & 0.073 \\
\hline 10 & Lead, $\mathrm{mg} / \mathrm{kg} \mathrm{Pb}$ & 0.430 & 0.432 & 0.430 & 0.431 \\
\hline
\end{tabular}

Table 3.10: Physico-chemical parameters for plant from polluted soil sample (amended)

\begin{tabular}{|c|c|c|c|c|c|c|c|c|c|}
\hline \multirow[t]{2}{*}{$\mathrm{S} / \mathrm{N}$} & \multirow[t]{2}{*}{ PARAMETER } & \multicolumn{4}{|c|}{$\begin{array}{l}\text { Plant from Pot B (7.5kgSoil } \\
\text { polluted with } 75 \mathrm{~g} \text { crude oil } \\
\quad+150 \mathrm{~g} \text { Cow dung) }\end{array}$} & \multicolumn{4}{|c|}{$\begin{array}{l}\text { Plant from Pot C (7.5kg Soil polluted with } 150 \mathrm{~g} \\
\text { crude oil }+150 \mathrm{~g} \text { Cow dung) }\end{array}$} \\
\hline & & $\mathrm{B}_{1}$ & $\mathrm{~B}_{2}$ & $\mathrm{~B}_{3}$ & Average & $\mathrm{C}_{1}$ & $\mathrm{C}_{2}$ & $\mathrm{C}_{3}$ & Average \\
\hline 1 & Moisture content, \% & 14.28 & 14.37 & 14.73 & 14.46 & 13.83 & 14.13 & 13.92 & 13.96 \\
\hline 2 & Ash content, \% & 15.48 & 15.37 & 15.87 & 15.57 & 15.89 & 15.76 & 15.66 & 15.77 \\
\hline 3 & Organic matter, \% & 78.28 & 76.38 & 79.47 & 78.04 & 76.93 & 77.04 & 77.33 & 77.10 \\
\hline 4 & $\begin{array}{l}\text { Nitrate-Nitrogen, } \\
\mathrm{mg} / \mathrm{kg} \mathrm{NO} \mathrm{N}_{3} \mathrm{~N}\end{array}$ & 8.94 & 9.02 & 8.94 & 8.97 & 8.76 & 9.94 & 8.34 & 9.01 \\
\hline 5 & Potassium, mg/kg K & 15.72 & 14.93 & 14.78 & 15.14 & 13.93 & 14.07 & 13.77 & 13.92 \\
\hline 6 & Calcium, mg/kg Ca & 11.10 & 10.92 & 10.98 & 11.00 & 11.07 & 10.70 & 10.38 & 10.72 \\
\hline 7 & Nickel, mg/kg Ni & 1.646 & 1.603 & 1.588 & 1.612 & 2.188 & 1.908 & 1.986 & 2.027 \\
\hline 8 & Mercury, mg/kg Hg & 1.908 & 2.007 & 2.111 & 2.009 & 2.807 & 3.104 & 3.012 & 2.974 \\
\hline 9 & Cadmium, mg/kg Cd & 0.016 & 0.029 & 0.012 & 0.019 & 0.027 & 0.034 & 0.022 & 0.028 \\
\hline 10 & Lead, mg/kg Pb & 3.750 & 3.738 & 3.750 & 3.746 & 4.081 & 3.828 & 3.857 & 3.922 \\
\hline
\end{tabular}

Table 3.11: Physico-chemical parameters for plant from polluted soil sample (unamended)

\begin{tabular}{|c|c|c|c|c|c|c|c|c|c|}
\hline \multirow[t]{2}{*}{$\mathrm{S} / \mathrm{N}$} & \multirow[t]{2}{*}{ PARAMETER } & \multicolumn{4}{|c|}{$\begin{array}{l}\text { Plant from Pot D (7.5kg Soil polluted } \\
\text { with } 75 \mathrm{~g} \text { crude oil) }\end{array}$} & \multicolumn{4}{|c|}{$\begin{array}{l}\text { Plant from Pot E (7.5kg Soil polluted with } \\
\text { 150g crude oil) }\end{array}$} \\
\hline & & $\mathrm{D}_{1}$ & $\mathrm{D}_{2}$ & $\mathrm{D}_{3}$ & Average & $\mathrm{E}_{1}$ & $\mathrm{E}_{2}$ & $\mathrm{E}_{3}$ & Average \\
\hline 1 & Moisture content, \% & 13.36 & 14.08 & 13.82 & 13.75 & 11.16 & 11.44 & 10.94 & 11.18 \\
\hline 2 & Ash content, $\%$ & 15.88 & 15.29 & 14.85 & 15.34 & 15.64 & 15.37 & 15.38 & 15.46 \\
\hline 3 & Organic matter, \% & 75.12 & 76.64 & 76.11 & 75.96 & 73.84 & 75.02 & 74.93 & 74.60 \\
\hline 4 & $\begin{array}{l}\text { Nitrate-Nitrogen, } \\
\mathrm{mg} / \mathrm{kg} \mathrm{NO} \mathrm{N}_{3}-\mathrm{N}\end{array}$ & 7.97 & 7.58 & 7.86 & 7.80 & 7.86 & 7.83 & 7.94 & 7.88 \\
\hline 5 & Potassium, mg/kg K & 13.63 & 13.85 & 14.03 & 13.84 & 13.22 & 13.70 & 13.28 & 13.40 \\
\hline 6 & Calcium, mg/kg Ca & 12.73 & 11.70 & 11.85 & 12.09 & 11.07 & 10.70 & 10.38 & 10.72 \\
\hline 7 & Nickel, mg/kg Ni & 1.583 & 1.701 & 1.685 & 1.656 & 2.328 & 2.448 & 2.392 & 2.389 \\
\hline 8 & Mercury, mg/kg Hg & 2.204 & 2.187 & 2.417 & 2.269 & 2.811 & 2.920 & 3.144 & 2.958 \\
\hline 9 & Cadmium, mg/kg Cd & 0.022 & 0.026 & 0.023 & 0.024 & 0.027 & 0.034 & 0.022 & 0.028 \\
\hline 10 & Lead, mg/kg Pb & 3.864 & 3.868 & 3.899 & 3.877 & 3.998 & 3.897 & 4.126 & 4.007 \\
\hline
\end{tabular}




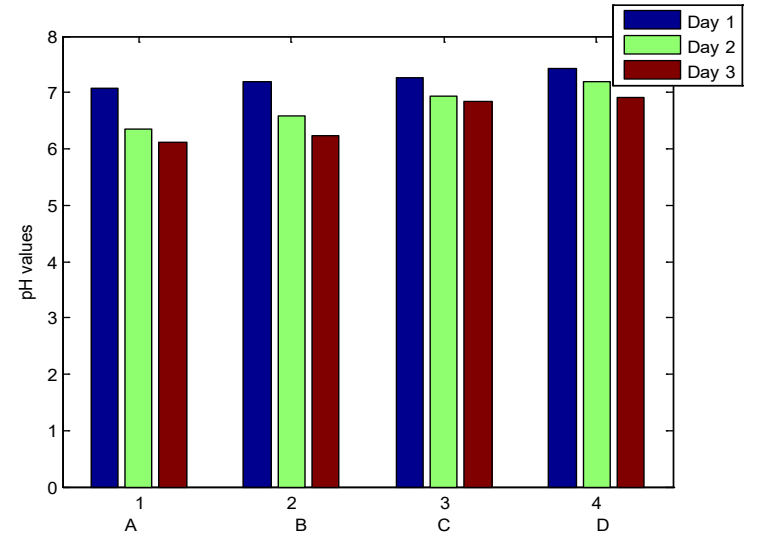

Figure 3.1: Comparison of $\mathrm{pH}$ value for the different samples for the three-time period

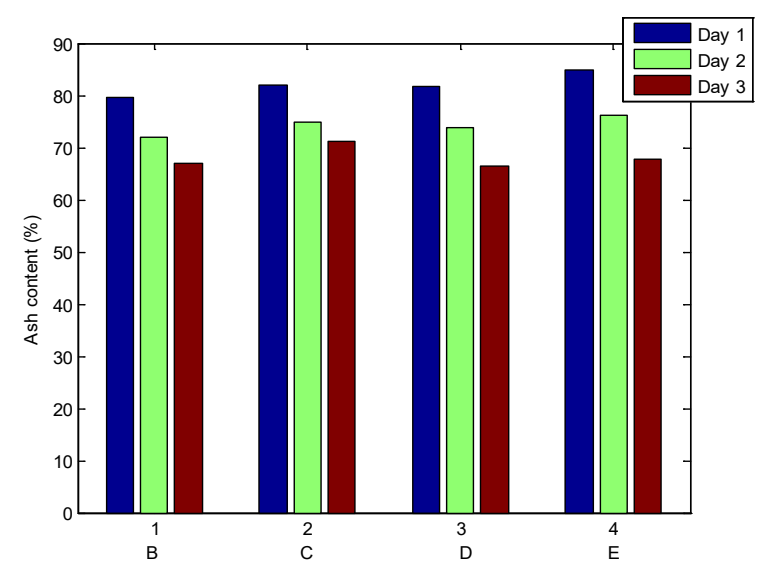

Figure 3.3: Comparison of ash content for the different samples for the three-time period

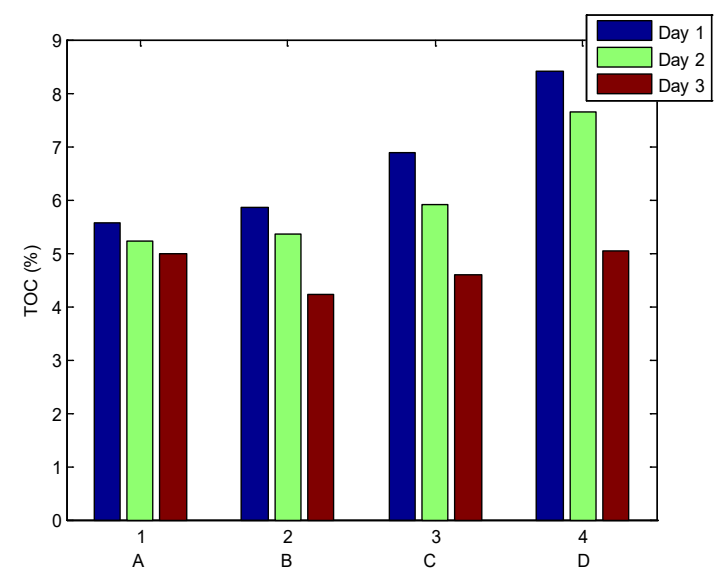

Figure 3.5: Comparison of total organic carbon for the different samples for the three-time period

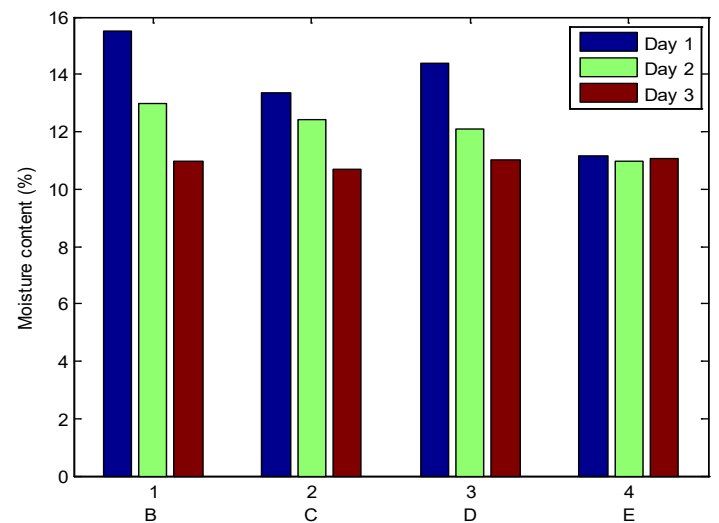

Figure 3.2: Comparison of moisture content for the different samples for the three-time period

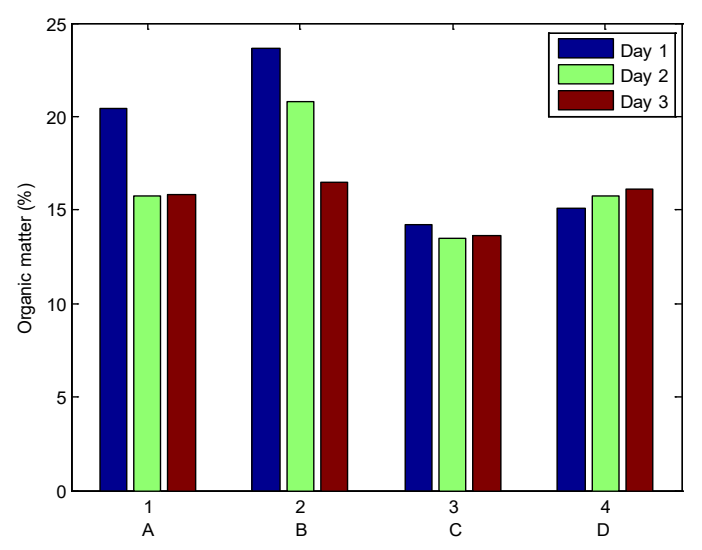

Figure 3.4: Comparison of organic matter for the different samples for the three-time period

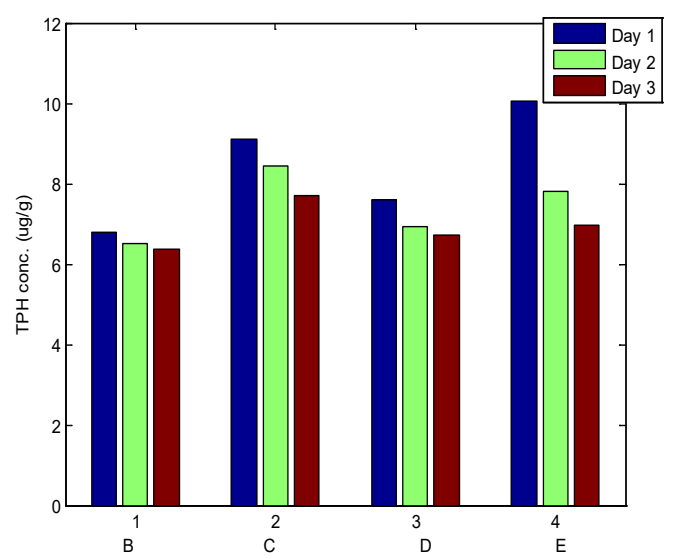

Figure 3.6: Comparison of total petroleum hydrocarbon for the different samples for the three-time period 


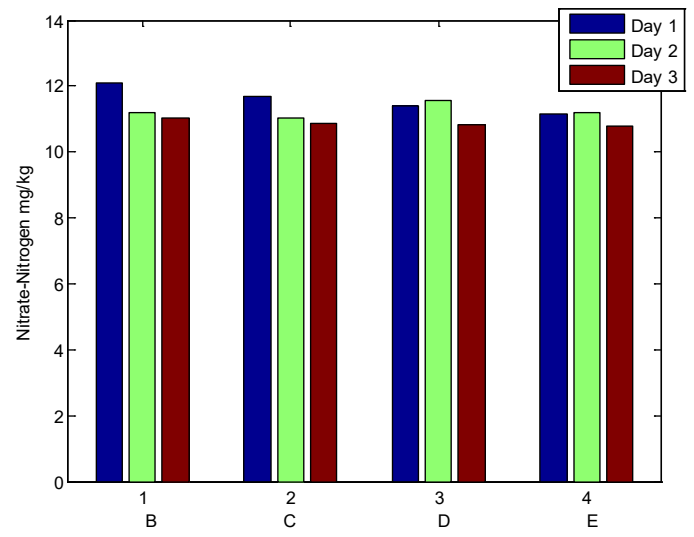

Figure 3.7: Comparison of nitrate-nitrogen for the different samples for the three-time period

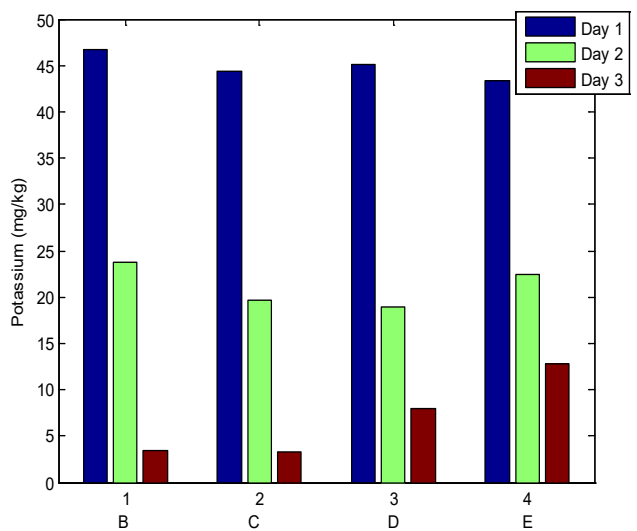

Figure 3.9: Comparison of potassium for the different samples for the three-time period

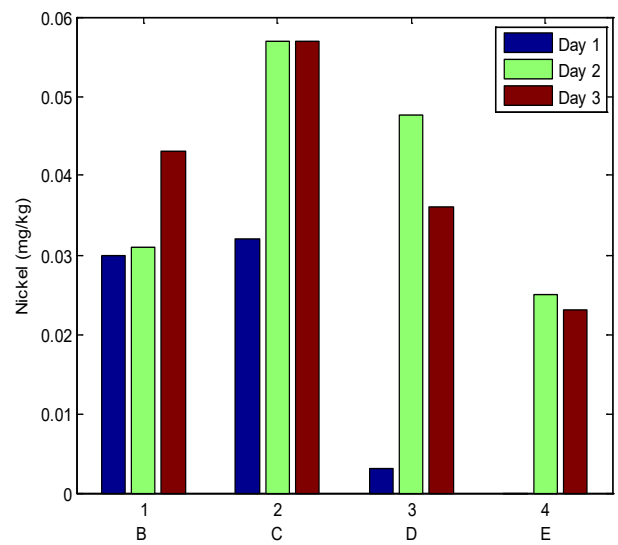

Figure 3.11: Comparison of nickel for the different samples for the three-time period

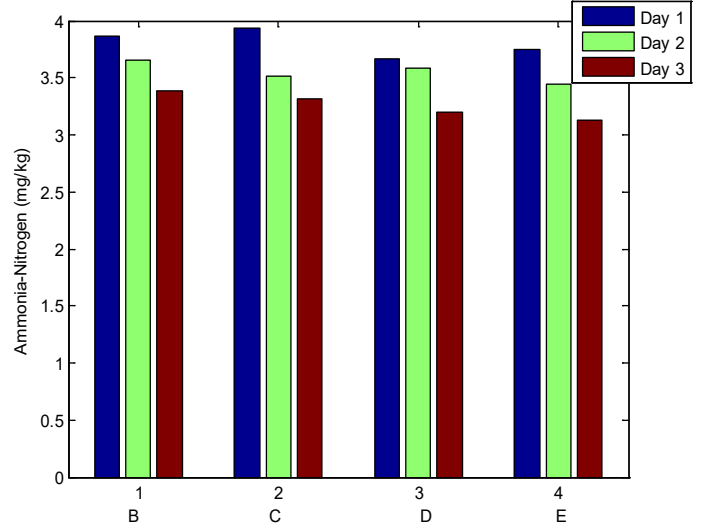

Figure 3.8: Comparison of ammonia-nitrogen for carbon for the different samples for the three-time period

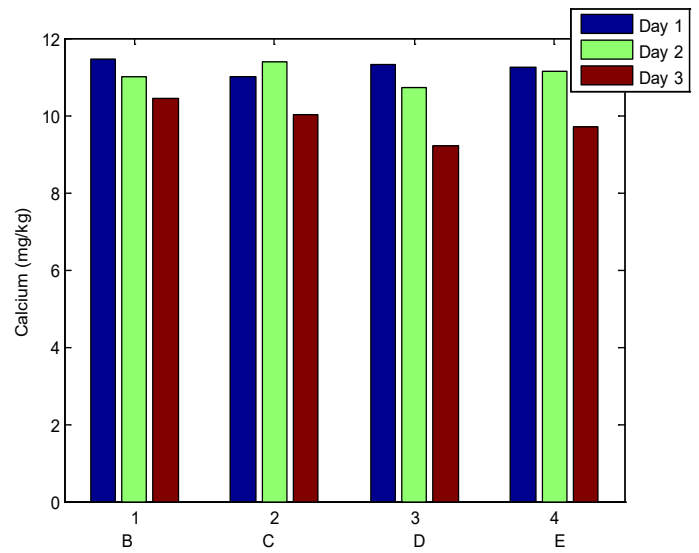

Figure 3.10: Comparison of calcium for the different samples for the three-time period

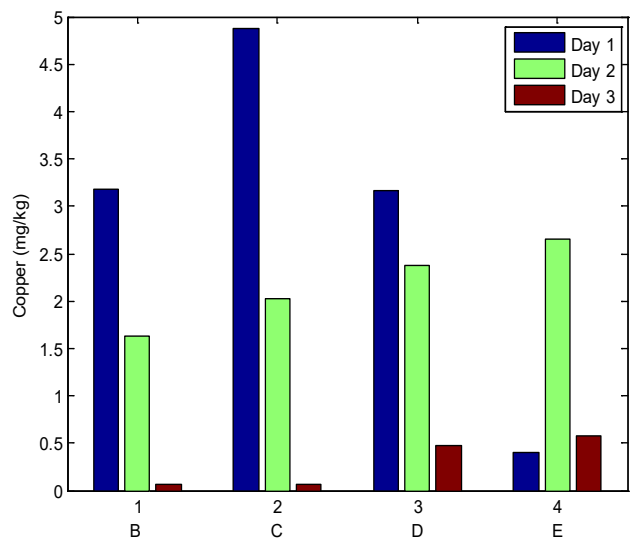

Figure 3.12: Comparison of cupper for the different samples for the three-time period 


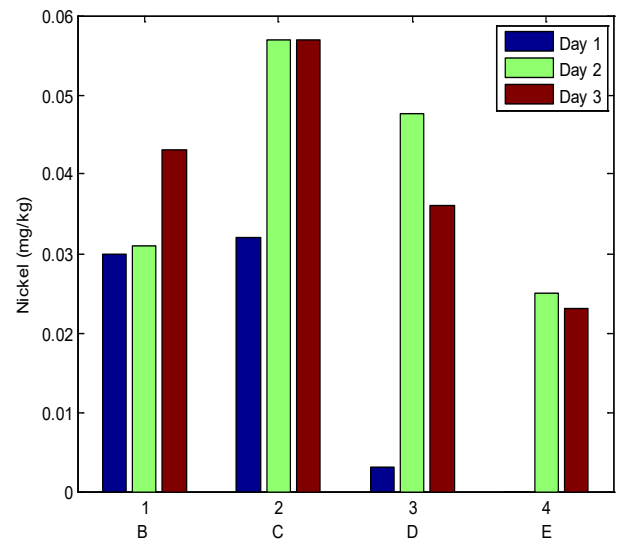

Figure 3.13: Comparison of nickel for the different samples for the three-time period

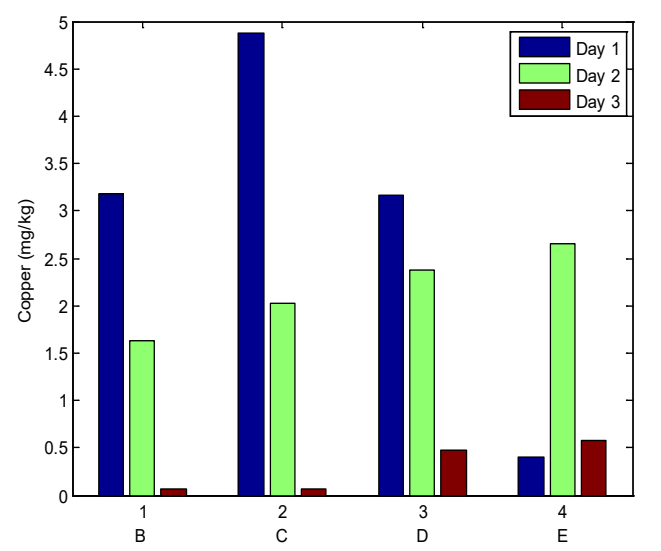

Figure 3.14: Comparison of cupper for the different samples for the three-time period

\subsection{DISCUSSION OF RESULTS}

The experimental results obtained for the four soil samples were compared for the three different periods the samples were monitored for phytoremediation. The results show the different components behavior of the soil at the intervals for the four samples considered (i.eB, C, D and E).

The result for $\mathrm{pH}$ values for the different samples for the varied time periods were collected is shown in Figure 3.1. For all results, there is reduction in the $\mathrm{pH}$ value as time progresses. The result showed that the soil becomes more acidic as time progresses for all sample mixture. For samples B and C, there is an initial huge decrease in the $\mathrm{pH}$ values which is followed by gradual decrease in the values.

In figure 3.2, there is a significant change in the moisture content when cow dung is added for both $75 \mathrm{~g}$ and $150 \mathrm{~g}$ contaminated soil. As time progressed, moisture contents of $\mathrm{B}, \mathrm{C}$ and $\mathrm{D}$ decreased continually while $\mathrm{E}$ had no significant change. A huge reduction was seen at the first check for B and D, which follows with a gradual reduction in the moisture content.

The ash content of the soil as shown in figure 3.3 shows a very little decrease with the addition of cow dung for both the $75 \mathrm{~g}$ and $150 \mathrm{~g}$ crude oil contaminations. All samples showed a progressive decrease in the value of the ash content with time though the degradation was rapid in soil samples E and D.

The comparison for the different samples for the organic matter is shown in figure 3.4. For soil samples B and $\mathrm{D}$, the organic matter dropped to a certain level and remained almost constant, though that of B had a very remarkable drop. $\mathrm{C}$ shows a higher value of initial organic matter and a high decrease in the values of the organic matter with time. E shows a gradual increase in the values of the organic matter with time. Generally, it can be seen that the addition of cow dung show a significant increase in the value of the organic matter

Percentage TOC values for the four samples are shown in figure 3.5. All samples showed a decrease in the value of the percentage TOC with time though C, D and E gave a higher decrease after the first-time interval. From the result gotten, it can clearly be seen that the values of the TOC dropped with the addition of cow dung.

The result for TPH concentration as seen in figure 3.6 gave a decrease in the values of TPH for all samples considered. The decrease for sample B was very minute as compared to sample D when cow dung was not added, and similar result was also noticed for $\mathrm{C}$ and $\mathrm{E}$. The addition of cow dung reduced the concentration of the TPH in the soil for both $75 \mathrm{~g}$ and $150 \mathrm{~g}$ crude oil contaminations.

Irregular behavior was seen in the values of nitrate-nitrogen for samples $\mathrm{D}$ and $\mathrm{E}$ as shown in figure 3.7, but a gradual decrease in the addition of cow dung for B and C. For D and $\mathrm{E}$ the values increased for the first time analysis and dropped for the second time analysis. In general, the addition of cow dung increased the values of the nitratenitrogen.

For the ammonia-nitrogen value as shown in Figure 3.8, there was a decrease in their values with time. Generally, there is a significant increase in the values of ammonia-nitrogen with the addition of cow dung.

There was a very high decrease in the potassium content of the soil with time, most especially with the addition of cow dung, as seen in Figure 3.9. There was no significant change in the initial values of the potassium with the addition of cow dung. 
Remediation Ability of Melon Grass in A Crude Oil Polluted Soil in A Tropical Region

The calcium values as shown in Figure 3.10 showed a decrease in calcium part from the value for sample B, which gave an increase in the first-time check and a decrease afterwards. Generally, there was no significant change in the value of calcium with the addition of cow dung.

Looking at Figure 3.11, there was a very significant increase in the value of nickel with the addition of cow dung, especially for the $150 \mathrm{~g}$ contaminated soil with crude oil as seen in sample C. Sample B showed a little increase in the value of nickel and a notable increase afterwards, while $C$ gave a very significant increase in the first-time check and constant afterwards. The same behavior was seen in D and $\mathrm{E}$ where there is an increment in the first-time check and decrease afterwards.

The copper values as seen in figure 3.12 showed a decrease in the values with time apart from E sample which gave an increase in the first-time check and a decrease afterwards. There is no significant change in the initial value of the sample with addition of cow dung for $75 \mathrm{~g}$ contaminated soil with crude oil, but a very significant increase when cow dung was added to $150 \mathrm{~g}$ contaminated soil with crude oil.

For the values of zinc, figure 3.13 shows that there was no significant change for the initial values when cow dung is added to the $150 \mathrm{~g}$ crude oil contaminated soil. There was decrease in the zinc content of soil samples as time progressed for all samples considered.

\section{CONCLUSSION}

The technology for phytoremediation that was employed in this study is a simple, effective, inexpensive and environmentally friendly approach, whose bio-stimulant is readily available, cheap and is compatible with the environment.

These observations indicate that the cow dung (animal source waste) used significantly enhanced phytodegradation in soil. Similar observations have been reported for the use of plant and animal-derived organic waste in the bioremediation of soil contaminated with petroleum hydrocarbons. Liu et al. (2009) used organic manure made up of rice straw and pig dung to bio-stimulate the degradation of an oily sludge and obtained a TPH reduction of 58.2\% in a remediation period of 360 days, while Agarry et al. (2013) in their investigation on kinetic model and half-life study of Bonny light crude oil amended with crop residue and animal derived organic manure confirms that the use of crop residue and animal derived organic manure improved the rate of biodegradation of hydrocarbon in a crude oil contaminated soil.

\section{SOURCES OF FUNDING}

None.

\section{CONFLICT OF INTEREST}

None.

\section{ACKNOWLEDGMENT}

None.

\section{REFERENCES}

[1] Abioye, P.O., Abdul-Aziz A. \& Agamuthu, P. (2009). Enhanced biodegradation of used engine oil in soil amended with organic wastes. Water Air Soil Poll., 209 (1): (173-179).

[2] Agarry, S.E. \&Lukuman, J.A. (2013). Application of Carbon-Nitrogen Supplementation from Plant and Animal Sources in In-situSoil Bioremediation of Diesel Oil: Experimental Analysis and Kinetic Modeling; Journal of Environment and Earth Science; Vol. 3, No.7.

[3] Agarry, S.E., Aremu, M.O. \&Aworanti O.A. (2013). Kinetic modelling and half- life study on enhanced soil bioremediation of bonny light crude oil amended with crop andanimal-derived organic waste. J Pet Environ Biotechnol 4: 137. 
Uwazie M.C., Obijiaku J.C., Onukwuli O.D., Babayemi A.K, and Umeuzuegbu J.C

[4] Ana, O.E. (2000). Remediation potential of petroleum refinery waste contaminated land at AlesaEleme, Rivers State. M.Eng Thesis: University of Port Harcourt.

[5] Aronson, D., Boethling, R. \& Howard P. (2000). Estimating biodegradation half-lives for use in chemical screening. Chemosphere.63: 1953-1960.

[6] Ayotamuno, M.J., Kogbara R.B.\&Probert S.D. (2005). Bioremediation of a crude oil polluted agricultural soil at Port-Harcourt, Nigeria. School of Engineering Cranfield University, Bedfordshire, UK.

[7] Choronn, M. (2010). Bioremedition of crude oil pollutted soil by the application of fertilizers. Iran J Environ Health SciEng 7:319-326.

[8] Cunningham, C.J. \& Philip, J.C. (2000). Comparison of Biostimulation in ex-situ Treatment of Diesel Contaminated Soil,

[9] Dadrasania, B.N. (2013). The potential use of biowaste (potato skin, soy cake, cow dung) to remediate a diesel fuel contamianated soil, Science Publishers Inc. pp.34-39.

[10] Ebere, J.U., Wokoma, E.C., Wokocha, C.C. (2011). Enhanced remediation of a hydrocarbon polluted soil, Research journal of environment and earth science.

[11] Ijah, J. \&Antal, S.P. (2003). The Potential Use of Poultry drop microorganisms for all Spill Remediation. Environ 2389-95.

[12] Ikhajiagbe, B. \&Anoliefo, G.O. (2011). Natural attenuation of a 14-month-old waste engine oil polluted soil. J Soil Sci Environ Manage 2: 184-192.

[13] Leahy, J.G. \& Crowell, R. R. (1990). Microbial Degradation of Hydrocarbons in theEnvironment. Microbio.Rev. 3, 305.

[14] Liu, P.G., Tsung, C.C., Liang-Ming, W., Chun-Hsuan, K., Po-Tseng, P. \& Sheng-Maletic, S. (2009). Degradation kinetics of an aged hydrocarbon contaminated soil. Water Air Soil Poll 202:149-159.

[15] Morel, J.L., Echevarria, G. \&Goncharova, N. (Eds.) (2002). Phytoremediation of Metal-Contaminated Soils, IOS Press, Amsterdam, and Springer in conjunction with the NATO Public Diplomacy Division - Springer.

[16] Okieimen, C.O. \&Okiemen, F.E. (2005). Bioremediation of crude polluted soil-effect of poultry droppings and natural rubber processing sludge application on biodegradation of petroleum hydrocarbon. Environ Sci.12:18.

[17] Okolo, J.C., Amadi, E.N. \&Odu,T.I. (2005). Effects of Soil Treatments using animalmanure on Crude Oil contaminated sandy-loam soil. Appl. Ecol. Environ. Res. 47-53.

[18] Onifade, A. K. (2007). Bioremediation of Crude Oil Polluted Soil in the Niger-Delta Area of Nigeria Using Enhanced Natural Attenuation. Res. J. Appal. Sci. 2498-504.

[19] Osoka, E.C.\&Onyelucheya,O.E. (2011). Data-Driven Model for Palm Bunch Ash-Enhanced Bioremediation of a crude oil contaminated soil, 362-363.

[20] Oyoh, K.B \&Osoka, E.C. (2002). Rate Model for Bioremediation Based on Total Petroleum Hydrocarbon Content, Journal of Nigerian Society of Chemical Engineers, 22 (2007),50-56.

[21] Parales, R.E., Bruce, C.N., Schmid, A. \&Wackett, L. P. (2003). Biodegradation, biotransformation, and biocatalysis (B3). Applied and Environmental Microbiology, 68(10), 4699-4709.

[22] Vidali, M. (2001). Bioremediation: An overview. J. Appl. Chemistry, 73(7), 1163-1172.

[23] Zahed, M.A. (2011). Kinetic modeling and half-life study on bioremediation of crude oil dispersed by corexit 9500. J Hazard Mater 185:1027-1031. 